\title{
The role of telocytes in morphogenetic bioelectrical signaling: once more unto the breach
}

\author{
Lawrence Edelstein $^{1 *}$ and John Smythies ${ }^{2}$ \\ ${ }^{1}$ Medimark Corporation, Del Mar, CA, USA \\ 2 Department of Psychology, Center for Brain and Cognition, University of California, San Diego, La Jolla, CA, USA \\ *Correspondence: larry.edelstein@claustrum.com
}

Edited by:

Jochen C. Meier, Max Delbrück Center for Molecular Medicine, Germany

Reviewed by:

Laurentiu M. Popescu, Victor Babeş National Institute of Pathology, Romania

Viorel Pais, National Institute of Neurology and Neurovascular Diseases, Romania

Keywords: morphogenetic code, bioelectrical signaling, telocytes, gap junctions, information processing, Vmems, network dynamics, stem cells

\section{INTRODUCTION}

This paper explores the possibility that telocytes play a major role in morphogenetic bioelectrical signaling.

\section{THE FACTS}

In a series of recent papers, Levin and his coworkers (Levin, 2009, 2012; Adams and Levin, 2012; Tseng and Levin, 2012, 2013) introduced the concept of a morphogenetic code based on bioelectrical signaling between cells. They showed that patterns of resting potentials (Vmem) in non-excitable cells act as instructive signals during embryogenesis, regeneration and cancer suppression in a wide range of tissues. They state that bioelectrical signaling among non-excitable cells, coupled by gap junctions, simulates neural network-like dynamics and underlies the information-processing functions required by complex pattern formation in vivo which result in coherent changes in anatomy. They identified one intermediate step between a specific bioelectric signal and altered cell anatomy as the regulation of small signaling molecules through transporters. Furthermore, they showed that the particular morphological change induced is dependent only on the voltage itself and not on the genetic identity of the channel, nor on the chemical species of the ion involved. Evidence from embryology suggests that narrow ranges of transmembrane potential specify the growth of specific anatomical structures such as an eye, head or tail. The authors suggest that this system may form a code, given that each cell has not one but many domains of Vmem along its surface, and so the spatial distribution of voltage values could form a rich combinatorial code. Vmem gradients form an important signal for modulating stem cell proliferation and differentiation (Pai and Levin, 2014). The authors conclude “... our hypothesis is that the existing highly successful theoretical apparatus for information processing in neurobiology could be extended to understand the properties of highly dynamic, self-repairing tissues and organs... we wondered whether in some cases, the bioelectric signal also activates a positional information pathway that guides the induced growth toward the locally-appropriate anatomical identity."

\section{OUR HYPOTHESIS}

We will present the case that telocytes (TCs) may play an essential role in morphogenetic bioelectrical signaling. In previous papers we have reviewed the key role that TCs may play in morphogenesis in nearly all organs in the body (Smythies and Edelstein, 2013; Edelstein and Smythies, 2014). Telocytes are characterized by having very small cell bodies consisting of a nucleus and a small amount of cytoplasm, and "extremely long and thin" tubular processes called telopodes up to 100 micrometers long, yet only 20-200 nanometers wide (Popescu and Faussone-Pellegrini, 2010; for the most comprehensive review of the literature see www.telocytes.com). The caliber of the telopodes is not uniform, which possess very thin podomers and dilations named podoms (Gherghiceanu and Popescu, 2012). They make synapses of various kinds, including adhaerens contacts and gap junctions, with practically every other type of cell in the tissue, including blood vessels, nerve fibers, fibroblasts, muscle cells, immune cells, glandular cells, stem cells and also other TCs. Thus, they form a continuous net wrapped around the larger cells of the organ. They exhibit a wide range of functions. The present consensus is that TCs could form an extensive intercellular information transmission and executive system that may utilize electric currents, small molecules, exosomesand possibly electrical events within the cytoskeleton-to modulate homeostasis, stem cell activity, tissue repair, peristalsis, anticancer activity and other complex functions in many organs (Gherghiceanu and Popescu, 2010; Popescu et al., 2011; Cretoiu et al., 2012a,b,c, 2013; Popescu et al., 2012; Luesma et al., 2013). These activities include extensive morphogenetic tissue repair after injuries such as cardiac ischemia and stroke in which the recruitment of stem cells plays a major role (Manole et al., 2011; Zhao et al., 2013).

Telocytes are also involved in the electrical modulation of excitable tissue, such as the smooth muscle of the gut and uterus. They are reported as being capable of spontaneously initiating electrical activity, which can be modulated by the stimulation of enteric nerves (Zhu et al., 2013); this process involves calcium transients (Yamashita, 2010). Cretoiu et al. (2013) reported the existence of hyperpolarization-activated chloride-inward current with calcium dependence and the absence of L-type calcium channels in human myometrium. Small-conductance calcium-activated 
potassium (SK3) channels have been detected in TCs in human myometrium wherein they reduce contractility (Rosenbaum et al., 2012). There is also evidence that they may mediate cholinergic neuromuscular transmission in ileal smooth muscle (Tanahashi et al., 2013). Sheng et al. (2014) examined human cardiac-cultured TCs and found that these cells expressed large conductance $\mathrm{Ca}(2+)$-activated $\mathrm{K}(+)$ current $(\mathrm{BKCa})$ and inwardly rectifying $\mathrm{K}(+)$ current (IKir), but not transient outward $\mathrm{K}(+)$ current (Ito) and ATP-sensitive potassium current (KATP). Other TC functions include modulation of glandular and immune activity. The reader is referred to our previous papers for further details.

We suggest that the TC system is well equipped to form a major part of the bioelectric "information pathway" postulated by Levin (2012) between cells in most tissues. The system may operate as follows: Since the TC net makes gap junction contacts with the cells in an organ its electrical activity could modulate, and be modulated by, the individual Vmems of these cells. Furthermore, the TC net could support wide-reaching patterns of the network dynamics postulated by Levin (2012). This in turn could modulate all of the other functions exerted by the TC net including morphogenesis, cancer control, as well as tissue repair and remodeling functions. In a recent review of gap junctions, Shimizu and Stopfer (2013) stated that their properties can be complex and surprising. Gap junctions help generate, propagate, and regulate neural oscillations, filter electrical signals, and can be modulated in a variety of ways. However, it is possible that a system of information transfer "A" between cells, which depends only upon direct sequential cell-to-cell gap junctions, might be less efficient than a system " $\mathrm{B}$ " such as the TC net, which is wholly devoted to the broad and rapid transmission of information via gap junctions. Furthermore, the fast integration of Vmem information with other sources of and targets for - this information would be harder for " $\mathrm{A}$ " than for "B." As well, the TC net provides a mechanism for the immediate coordinated recruitment of stem cells that system "A" so far lacks.

We therefore posit that the universally distributed, electrically active, gap-junction linked, morphogenetic, cancer-suppressing and stem cellcontrolling TC network supplements, but does not replace, direct cell-to-cell systems in bioelectrical signaling.

\section{REFERENCES}

Adams, D. S., and Levin, M. (2012). Endogenous voltage gradients as mediators of cell-cell communication: strategies for investigating bioelectrical signals during pattern formation. Cell Tissue Res. 352, 95-122. doi: 10.1007/s00441-012-1329-4

Cretoiu, D., Cretoiu, S. M., Simionescu, A. A., and Popescu, L. M. (2012a). Telocytes, a distinct type of cell among the stromal cells present in the lamina propria of jejunum. Histol Histopathol. 27, 1067-1078. Available online at: http://www.hh.um.es/Abstracts/Vol_27/27_8/27_8 _1067.htm

Cretoiu, S. M., Cretoiu, D., Marin, A., Radu, M., and Popescu, L. (2013). Telocytes: 221 ultrastructural, immunohistochemical and electrophysiological characteristics in 222 human myometrium. Reproduction 12:223. doi: 10.1530/REP-12-0369

Cretoiu, S. M., Cretoiu, D., and Popescu, L. M. (2012b). Human myometrium - the ultrastructural 3D network of telocytes. J. Cell. Mol. Med. 16, 2844-2849. doi: 10.1111/j.15824934.2012.01651.x

Cretoiu, S. M., Simionescu, A., and Popescu, L. M. (2012c). Telocytes in human fallopian tube and uterus express estrogen and progesterone receptors. Sex Steroids 217, 91-114. doi: 10.5772/25978

Edelstein, L., and Smythies, J. (2014). Epigenetic aspects of telocytes/cordocytes: jacks of all trades, masters of most. Front. Cell. Neurosci. 8:32. doi: 10.3389/fncel.2014.00032

Gherghiceanu, M., and Popescu, L. M. (2010). Cardiomyocyte precursors and telocytes in epicardial stem cell niche: electron microscope images. J. Cell. Mol. Med. 14, 871-877. doi: 10.1111/j.15824934.2010.01060.x

Gherghiceanu, M., and Popescu, L. M. (2012). Cardiac telocytes - their junctions and functional implications. Cell Tissue Res. 348, 265-279. doi: 10.1007/s00441-012-1333-8

Levin, M. (2009). Bioelectric mechanisms in regeneration: unique aspects and future perspectives. Semin. Cell Dev. Biol. 20, 543-556. doi: 10.1016/j.semcdb.2009.04.013

Levin, M. (2012). Molecular bioelectricity in developmental biology: new tools and recent discoveries: control of cell behavior and pattern formation by transmembrane potential gradients. Bioessays 34, 205-217. doi: 10.1002/bies.201100136

Luesma, M. J., Gherghiceanu, M., and Popescu, L. M. (2013). Telocytes and stem cells in limbus and uvea of mouse eye. J. Cell. Mol. Med. 17, 1016-1024. doi: $10.1111 / \mathrm{jcmm} .12111$

Manole, C. G., Cismaşiu, V., Gherghiceanu, M., and Popescu, L. M. (2011). Experimental acute myocardial infarction: telocytes involvement in neoangiogenesis. J. Cell. Mol. Med. 15, 2284-2296. doi: 10.1111/j.1582-4934.2011.01449.x

Pai, V. P., and Levin, M. (2014). "Bioelectric controls of stem cell function," in Stem Cells: From Basic Research to Therapy, eds F. Calegari and C. Waskow (New York, NY: CRC Press), 106-145.
Popescu, B. O., Gherghiceanu, M., Kostin, S., Ceafalan, L., and Popescu, L. M. (2012). Telocytes in meninges and choroid plexus. Neurosci. Lett. 516, 265-269. doi: 10.1016/j.neulet.2012. 04.006

Popescu, L. M., and Faussone-Pellegrini, M. S. (2010). TELOCYTES - a case of serendipity: the winding way from Interstitial Cells Of Cajal (ICC), via Interstitial Cajal-Like Cells (ICLC) to TELOCYTES. J. Cell. Mol. Med. 14, 729-740. doi: 10.1111/j.1582-4934.2010.01059.x

Popescu, L. M., Manole, E., Şerboiu, C. S., Manole, C. G., Suciu, L. C., Gherghiceanu, M., et al. (2011). Identification of telocytes in skeletal muscle interstitium: implication for muscle regeneration. J. Cell. Mol. Med. 15, 1379-1392. doi: 10.1111/j.1582-4934.2011.01330.x

Rosenbaum, S. T., Svalø, J., Nielsen, K., Larsen, T., Jørgensen, J. C., and Bouchelouche, P. (2012). Immunolocalization and expression of smallconductance calcium-activated potassium channels in human myometrium. J. Cell. Mol. Med. 16, 3001-3008. doi: 10.1111/j.1582-4934.2012. 01627.x

Sheng, J., Shim, W., Lu, J., Lim, S. Y., Ong, B. H., Lim, T. S., et al. (2014). Electrophysiology of human cardiac atrial and ventricular telocytes. J. Cell. Mol. Med. 18, 355-362. doi: 10.1111/jcmm. 12240

Shimizu, K., and Stopfer, M. (2013). Gap junctions. Curr. Biol. 23, R1026-R1031. doi: 10.1016/j.cub. 2013.10.067

Smythies, J., and Edelstein, L. (2013). Telocytes, exosomes, gap junctions and the cytoskeleton: the makings of a primitive nervous system? Front. Cell Neurosci. 7:278. doi: 10.3389/fncel.2013.00278

Tanahashi, Y., Ichimura, Y., Kimura, K., Matsuyama, H., Iino, S., Komori, S., et al. (2013). Cholinergic neuromuscular transmission mediated by interstitial cells of cajal in the myenteric layer in mouse ileal longitudinal smooth muscles. Naunyn Schmiedebergs Arch. Pharmacol. 387, 377-388. doi: 10.1007/s00210-013-0944-2

Tseng, A. S., and Levin, M. (2012). Transducing bioelectric signals into epigenetic pathways during tadpole tail regeneration. Anat. Rec. (Hoboken). 295, 1541-1551. doi: 10.1002/ar.22495

Tseng, A. S., and Levin, M. (2013). Cracking the bioelectric code: probing endogenous ionic controls of pattern formation. Commun. Integr. Biol. 6:e22595. doi: 10.4161/cib.22595

Yamashita, M. (2010). Synchronization of Ca2+ oscillations: a capacitative (AC) electrical coupling model in neuroepithelium. FEBS J. 277, 293-299. doi: 10.1111/j.1742-4658.2009.07439.x

Zhao, B., Chen, S., Liu, L., Yuan, Z., Qi, X., Quin, J., et al. (2013). Cardiac telocytes were decreased during myocardial infarction and their therapeutic effects for ischaemic heart in rat J. Cell. Mol. Med. 17, 123-133. doi: 10.1111/j.1582 4934.2012.01655.x

Zhu, Y. F., Wang, X. Y., Lowie, B. J., Parsons, S., White, L., Kunze, W., et al. (2013). Enteric sensory neurons communicate with interstitial cells of cajal to affect pacemaker activity in the small intestine. Pflugers Arch. doi: 10.1007/s00424-013-1374-1. [Epub ahead of print].

Conflict of Interest Statement: The authors declare that the research was conducted in the absence of any 
commercial or financial relationships that could be construed as a potential conflict of interest.

Received: 20 March 2014; accepted: 22 April 2014; published online: 13 May 2014.

Citation: Edelstein L and Smythies J (2014) The role of telocytes in morphogenetic bioelectrical signaling: once more unto the breach. Front. Mol. Neurosci. 7:41. doi: 10.3389/fnmol.2014.00041

This article was submitted to the journal Frontiers in Molecular Neuroscience.

Copyright (c) 2014 Edelstein and Smythies. This is an open-access article distributed under the terms of the Creative Commons Attribution License (CC BY).
The use, distribution or reproduction in other forums is permitted, provided the original author(s) or licensor are credited and that the original publication in this journal is cited, in accordance with accepted academic practice. No use, distribution or reproduction is permitted which does not comply with these terms. 